\title{
Managing the Attractive Magnetic Force between an Untethered Magnetically Actuated Tool and a Rotating Permanent Magnet
}

\author{
Arthur W. Mahoney, Samuel E. Wright, and Jake J. Abbott
}

\begin{abstract}
Untethered magnetic devices, such as magnetic microrobots and magnetically actuated capsule endoscopes, stand to significantly impact the field of minimally invasive medicine. These devices, which we refer to as magnetically actuated tools (MATs), are often controlled using electromagnets, which can be expensive to scale clinically. Due to their potential for low-cost and high strength, permanent magnets are being considered for MAT actuation. Great care must be taken for in vivo medical applications, however, as systems using single permanent magnets typically generate an attractive force, which may cause trauma if uncontrolled. In this paper, we present techniques for managing the attractive magnetic force. We find that in the case of rotating MATs actuated using a single rotating permanent magnet (RPM), for which magnetic torque is the primary form of actuation, the attractive magnetic force can be substantially reduced in magnitude and the timeaveraged attractive component eliminated when operating the MAT such that magnetic torque is maximized. We provide experimental demonstration that validates and illustrates the force management strategies presented.
\end{abstract}

\section{INTRODUCTION}

Untethered magnetic devices, such as magnetic microrobots [1] and magnetically actuated capsule endoscopes [2], have become an active area of research because of their potential impact to minimally invasive medicine. These devices derive their power from externally applied magnetic fields. Some forms of actuation apply magnetic force for pulling [3], [4], some apply magnetic torque for swimming through an open fluid or fluid-filled lumen [5] or rolling on a surface [6][10]. These devices are viewed as simple end-effectors of a larger robotic system, and we refer to them as magnetically actuated tools (MATs) with no implied size.

Magnetic fields used to actuate untethered MATs are typically produced using arrangements of electromagnets [1], [3], [11]. Because of their ability to generate clinically relevant magnetic fields at low cost, permanent magnet systems are gaining attention [4]-[8]. Electromagnetic systems can be designed to generate uniform magnetic fields (producing torque) and field gradients (producing force) that simplify MAT control. Permanent magnet systems generate nonuniform magnetic fields and field gradients, which significantly complicate control. When a MAT is stably actuated using a single permanent magnet, the generated magnetic force typically attracts the MAT in the direction of the permanent

This material is based upon work supported by the National Science Foundation under Grant Nos. 0952718 and 0654414

A. W. Mahoney is with the School of Computing, and S. E. Wright and J. J. Abbott are with the Department of Mechanical Engineering, University of Utah, Salt Lake City, UT, 84112 USA.

\{art.mahoney, jake.abbott\}@utah.edu

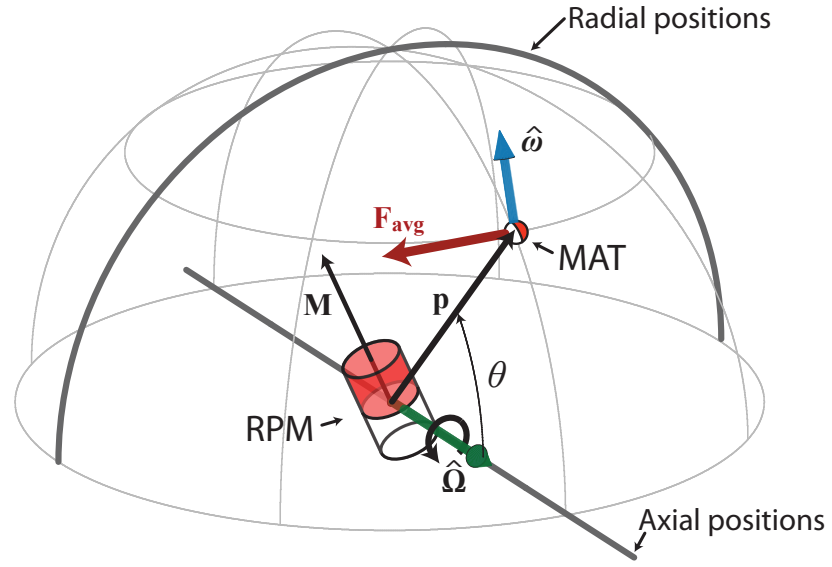

Fig. 1. If the RPM and MAT rotate about the $\hat{\boldsymbol{\Omega}}$ and $\hat{\boldsymbol{\omega}}$ axes, respectively, then the magnetic force $\mathbf{F}$ that attracts the MAT (positioned at $\mathbf{p}$ ) in the direction of the RPM can be dramatically reduced in magnitude when the MAT is actuated such that the applied magnetic torque is maximized. When actuated in this manner, the average magnetic force over one RPM revolution

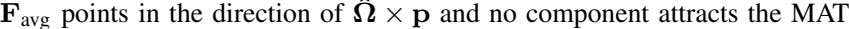
to the RPM. The angle between $\hat{\Omega}$ and $\mathbf{p}$ is measured by $\theta$.

magnet. This attractive force is useful if magnetic pulling assists or is the primary means of MAT locomotion. However, great care must be taken for in vivo medical applications, as an attractive force too large in magnitude may cause the MAT to pull toward the external magnet in an uncontrolled fashion, resulting in tissue deformation and potentially trauma.

Magnetic force can be decreased by increasing the distance between the MAT and the external magnet. However, increasing the distance comes at the cost of decreasing the applied magnetic field and the resulting torque that can be produced In the case of rotating MATs actuated using a single rotating permanent magnet (RPM), for which magnetic torque is the primary form of actuation, a decrease in torque can result in loss of control. Until recently, MATs actuated with a single RPM were exclusively operated in axial $\left(\theta=0^{\circ}\right.$ or $\left.180^{\circ}\right)$ or radial $\left(\theta=90^{\circ}\right)$ positions, where the behavior of the rotating magnetic field was easy to understand (Fig. 1). In these two positions, we have previously shown that the attractive magnetic force can be substantially decreased when the MAT is operated in a regime where the magnetic torque is maximized, without requiring the separation distance between the MAT and the RPM to increase [5], [6].

Recently, we have shown how to actuate rotating MATs in any position, eliminating the need to operate solely in axial or radial positions [7]. In this paper, we generalize the forcemanagement results of [5] and [6], which were originally 
developed exclusively for axial and radial positions, respectively, to any position. We find that if the MAT is actuated such that the magnetic torque is maximized in any position, the total instantaneous magnitude of the magnetic force can be substantially reduced without increasing the MAT-RPM separation distance, and simultaneously, the component of the time-averaged (i.e., net) magnetic force that attracts the MAT in the direction of the RPM can be entirely eliminated. The reduction of the attractive magnetic force is desirable for general safety, and also when the RPM position generates an attractive force that hinders MAT locomotion (e.g., when the desired MAT direction of travel is away from the RPM). Although it is not always beneficial to reduce the attractive magnetic force (e.g., when it assists desired MAT locomotion), this paper only considers its reduction. Understanding how to maximize the attractive-force contribution to MAT actuation remains for future work.

\section{Controlling Rotating MATs}

In this section, we summarize the results of [7] that are critical to this paper. All vectors are expressed in a common, static coordinate frame $\{\hat{\mathbf{x}}, \hat{\mathbf{y}}, \hat{\mathbf{z}}\}$, and the "hat" symbol denotes a vector normalized to unit length. Let $\mathbf{m}$ be the dipole moment of the MAT's magnetic body and let the MAT be position at $\mathbf{p}$ (measured relative to the RPM center). If a magnetic field $\mathbf{H}$ is externally applied to the MAT, then a magnetic torque is created and is given by $\boldsymbol{\tau}=\mu_{0} \mathbf{m} \times \mathbf{H}$, where $\mu_{0}$ is the permeability of free space. This torque causes $\mathbf{m}$ to rotate instantaneously in the direction of $\mathbf{H}$. If the magnetic field $\mathbf{H}$ rotates around an axis $\hat{\boldsymbol{\omega}}$, then $\boldsymbol{\tau}$ will cause the MAT to continuously rotate, which is typically converted into MAT propulsion via rolling or through screw propulsion. Regardless of the form of locomotion, the rotation axis of the MAT dipole moment $\mathbf{m}$ tends to align with the field rotation axis $\hat{\boldsymbol{\omega}}$ over time if possible. In this paper, we assume that the rotation axis of $\mathbf{m}$ is aligned with $\hat{\boldsymbol{\omega}}$.

If the applied magnetic field $\mathbf{H}$ is generated by a single RPM, then the magnetic field generated at the MAT position p can be approximated by the point-dipole model:

$$
\mathbf{H}=\frac{1}{4 \pi|\mathbf{p}|^{3}}\left(3 \hat{\mathbf{p}} \hat{\mathbf{p}}^{\top}-\mathbb{I}\right) \mathbf{M}=\frac{1}{4 \pi|\mathbf{p}|^{3}} \mathbb{H} \mathbf{M},
$$

where $\mathbf{M}$ is the RPM dipole moment, $\mathbb{I}$ is the identity matrix, and $\hat{\mathbf{p}}$ is the unit vector in the direction of $\mathbf{p}$. If the RPM is rotated with angular velocity $\Omega$, such that the RPM dipole moment $\mathbf{M}$ is always perpendicular to $\hat{\boldsymbol{\Omega}}$ (i.e., $\hat{\boldsymbol{\Omega}}^{\top} \mathbf{M}=0$ ) and the direction of rotation is found using a right-hand rule, then the applied field rotation axis $\hat{\omega}$ at position $\mathbf{p}$ is related to the RPM rotation axis $\hat{\Omega}$ by

$$
\hat{\boldsymbol{\omega}}=\widehat{\mathbb{H}^{-1} \hat{\boldsymbol{\Omega}}},
$$

where $\mathbb{H}^{-1}=(\mathbb{H}-\mathbb{I}) / 2$, requiring no matrix inversion.

As the RPM rotates about $\hat{\Omega}$, the instantaneous field magnitude $|\mathbf{H}|$ fluctuates in an elliptical fashion, which varies with $\mathbf{p}$ and is given by

$$
|\mathbf{H}|=\frac{|\mathbf{M}|}{4 \pi|\mathbf{p}|^{3}} \sqrt{1+3\left(\hat{\mathbf{M}}^{\top} \hat{\mathbf{p}}\right)^{2}} .
$$

The rotational velocity of $\mathbf{H}$ around $\hat{\boldsymbol{\omega}}$ also fluctuates instantaneously. If $|\boldsymbol{\omega}|$ and $|\boldsymbol{\Omega}|$ denote the instantaneous rotational velocities of $\mathbf{H}$ and the RPM, respectively, then $|\boldsymbol{\omega}|$ and $|\boldsymbol{\Omega}|$ are related by

$$
|\boldsymbol{\omega}|=\left(\frac{|\mathbf{H}|_{\min }|\mathbf{H}|_{\max }}{|\mathbf{H}|^{2}}\right)|\boldsymbol{\Omega}| .
$$

where $|\mathbf{H}|_{\min }$ and $|\mathbf{H}|_{\max }$ are the minimum and maximum values of $|\mathbf{H}|$ through one revolution of the RPM (the closedform solution of which can be found in [7]).

For rotating MATs, a common failure mode occurs when the MAT steps out of synchronization with the rotating local field. The rotation frequency above which the applied magnetic torque is too weak to keep the MAT synchronized with the rotating field is referred to as the "step-out" frequency and is denoted by $|\boldsymbol{\omega}|_{\text {so }}$. If a MAT's rotational inertia is negligible, then its angular velocity can be modeled as being proportional to the magnetic torque by a linear damping coefficient $c$, and $|\boldsymbol{\omega}|_{\text {so }}$ is the MAT rotational velocity at the maximum possible instantaneous magnetic torque $|\boldsymbol{\tau}|_{\max }$, which results in $|\boldsymbol{\omega}|_{\text {so }}=|\boldsymbol{\tau}|_{\max } / c$. The maximum instantaneous magnetic torque that can be produced on a MAT at position $\mathbf{p}$ is $|\boldsymbol{\tau}|_{\max }=\mu_{0}|\mathbf{m}||\mathbf{H}|$ (where $\mathbf{H}$ is given by (3)), making $|\boldsymbol{\omega}|_{\text {so }}=\mu_{0}|\mathbf{m}||\mathbf{H}| / c$. Rotating the RPM so that $|\boldsymbol{\omega}|=|\boldsymbol{\omega}|_{\text {so }}$ requires the maximum amount of available magnetic torque to remain synchronized, and as a result, causes the angle between the field $\mathbf{H}$ and the MAT dipole moment $\mathbf{m}$ (as $\mathbf{m}$ trails behind $\mathbf{H}$ ) to converge asymptotically to $90^{\circ}$.

\section{Minimizing Applied Magnetic Force}

Actuating MATs, using a single RPM in stable configurations, is made challenging by the presence of magnetic force that tends to attract the MAT in the direction of the RPM. The magnetic force $\mathbf{F}$ is a function of the MAT position $\mathbf{p}$, and the RPM and MAT dipole moments, $\mathbf{M}$ and $\mathbf{m}$, respectively. The magnetic force is given by $\mathbf{F}=\mu_{0} \nabla(\mathbf{H} \cdot \mathbf{m})$, which can be expressed as

$$
\mathbf{F}=\frac{3 \mu_{0}|\mathbf{m}||\mathbf{M}|}{4 \pi|\mathbf{p}|^{4}}\left[\begin{array}{l}
\hat{\mathbf{M}}^{\top} \mathbb{H}_{\mathrm{x}} \\
\hat{\mathbf{M}}^{\top} \mathbb{H}_{\mathrm{y}} \\
\hat{\mathbf{M}}^{\top} \mathbb{H}_{\mathrm{z}}
\end{array}\right] \hat{\mathbf{m}}=\kappa \mathbb{F} \hat{\mathbf{m}}
$$

where $\kappa=3 \mu_{0}|\mathbf{m}||\mathbf{M}| /\left(4 \pi|\mathbf{p}|^{4}\right)$ and where the symmetric matrix $\mathbb{H}_{\mathrm{x}}$ maps $\mathbf{m}$ to the spatial gradient of the field $\mathbf{H}$ in the $\hat{\mathrm{x}}$ direction and is given by

$$
\mathbb{H}_{\mathrm{x}}=\left(\hat{\mathbf{x}}^{\top} \hat{\mathbf{p}}\right)\left(\mathbb{I}-5 \hat{\mathbf{p}} \hat{\mathbf{p}}^{\top}\right)+\hat{\mathbf{x}} \hat{\mathbf{p}}^{\top}+\hat{\mathbf{p}} \hat{\mathbf{x}}^{\top} .
$$

The matrices $\mathbb{H}_{\mathrm{y}}$ and $\mathbb{H}_{\mathrm{z}}$ are defined similarly. The matrix $\mathbb{F}$ is symmetric and is a function of both $\hat{\mathbf{M}}$ and $\hat{\mathbf{p}}$. This implies that the direction of $\mathbf{F}$ is invariant to scaling $|\mathbf{p}|$ by moving the RPM nearer to or farther away from the MAT, provided the directions of $\hat{\mathbf{m}}, \hat{\mathbf{M}}$ and $\hat{\mathbf{p}}$ remain unchanged. Naturally, the magnitude of $\mathbf{F}$ scales with $|\mathbf{p}|^{-4}$. (Note that $\hat{\mathbf{m}}$ and $\hat{\mathbf{M}}$ can be interchanged in (5) without changing $\mathbf{F}$.)

The most conservative method for reducing the magnetic force is to produce an absolute upper bound on the maximum possible force magnitude $|\mathbf{F}|_{\text {max }}$ for any possible orientation 
of the MAT dipole moment $\mathbf{m}$ at the MAT position $\mathbf{p}$, and any orientation of the RPM dipole moment $\mathbf{M}$ over one RPM revolution. Safety can be guaranteed by increasing $|\mathbf{p}|$ so that $|\mathbf{F}|_{\text {max }}$ is sufficiently small. $|\mathbf{F}|_{\max }$ is found by maximizing the largest singular value of $\mathbb{F}$ subject to the constraint $\mathbf{M}^{\top} \hat{\boldsymbol{\Omega}}=0$ (because $\mathbf{M}$ rotates around $\hat{\boldsymbol{\Omega}}$ ), which produces

$$
|\mathbf{F}|_{\max }=\frac{\kappa}{2}\left(|\tilde{\mathbf{p}}|+\sqrt{4+5|\tilde{\mathbf{p}}|^{2}}\right) .
$$

where $\tilde{\mathbf{p}}=\left(\mathbb{I}-\hat{\mathbf{\Omega}} \hat{\Omega}^{T}\right) \hat{\mathbf{p}}$ is the projection of $\hat{\mathbf{p}}$ onto the plane perpendicular to $\hat{\Omega}$ (the plane in which $\mathrm{M}$ resides).

The maximum, worst-case force magnitude $|\mathbf{F}|_{\max }$ varies with both the magnitude and direction of p. Fig. 2 shows (7), normalized by $2 \kappa$, plotted as a function of the angle $\theta$, which measures the angle between $\hat{\mathbf{p}}$ and $\hat{\boldsymbol{\Omega}}$. From Fig. 2, it is clear that $|\mathbf{F}|_{\max }$ is half as strong in axial positions $\left(\theta=0^{\circ}\right.$ or $\left.180^{\circ}\right)$ as in radial positions $\left(\theta=90^{\circ}\right)$. For a chosen $\hat{\mathbf{p}},|\mathbf{F}|_{\max }$ can be reduced by increasing $|\mathbf{p}|$. In radial positions, $|\mathbf{p}|$ needs to be increased more than in axial positions to guarantee $|\mathbf{F}|_{\text {max }}$ falls below the same threshold. Although (7) is a conservative bound, a magnetic force nearly as large in magnitude as (7) is experienced by a MAT under normal operating conditions if the RPM is rotated slowly, in which case the magnetic torque always keeps the MAT dipole moment $\mathbf{m}$ nearly aligned with the rotating magnetic field $\mathbf{H}$ (i.e., $\hat{\mathbf{m}} \approx \hat{\mathbf{H}}$ ). The maximum magnetic force magnitude under this circumstance is plotted in Fig. 2 and is nearly identical to (7) in all MAT positions.

In any position, increasing $|\mathbf{p}|$ to limit the conservative bound (7) comes at the cost of decreasing $|\mathbf{H}|$ along with the potential magnetic torque $\tau$ that can be applied to the MAT. The RPM may lose control authority over the MAT if the magnetic torque is decreased too much. An approach for reducing the attractive force without sacrificing $|\mathbf{H}|$ and the potential magnetic torque is desirable. We find that this can be achieved by operating the MAT such that the applied magnetic torque $\tau$ is maximized, which occurs when the MAT is operated at its step-out frequency.

We assume that the MAT dipole moment $\mathbf{m}$ trails the applied field $\mathbf{H}$, and they both rotate around the axis $\hat{\boldsymbol{\omega}}$. Under this assumption, and because the MAT's dipole moment $\mathbf{m}$ is perpendicular to the field $\mathbf{H}$ at the MAT's step-out frequency, the direction of the MAT dipole moment $\hat{\mathbf{m}}$ can be found instantaneously:

$$
\hat{\mathbf{m}}=\hat{\mathbf{H}} \times \hat{\boldsymbol{\omega}}=\frac{\mathbf{H}}{|\mathbf{H}|} \times \frac{\mathbb{H}^{-1} \hat{\boldsymbol{\Omega}}}{\left|\mathbb{H}^{-1} \hat{\mathbf{\Omega}}\right|} .
$$

Substituting (8) into (5) and grouping terms produces the equation for the magnetic force when $\tau$ is maximized:

$$
\begin{array}{r}
\mathbf{F}=\frac{\kappa}{|\mathbf{H}|\left|\mathbb{H}^{-1} \hat{\mathbf{\Omega}}\right|}\left(3|\tilde{\mathbf{p}}|\left(\hat{\mathbf{M}}^{\top} \hat{\mathbf{p}}\right)^{2} \hat{\mathbf{d}}-\left(\hat{\mathbf{M}}^{\top} \hat{\mathbf{p}}\right) \hat{\mathbf{M}}_{\perp}\right. \\
\left.-\left(\hat{\mathbf{M}}^{\top} \hat{\mathbf{p}}\right)\left(\hat{\mathbf{M}}_{\perp}^{\top} \hat{\mathbf{p}}\right) \hat{\mathbf{p}}+2\left(\hat{\mathbf{M}}_{\perp}^{\top} \hat{\mathbf{p}}\right) \hat{\mathbf{M}}\right)
\end{array}
$$

where $\hat{\mathbf{d}}=\widehat{\hat{\mathbf{\Omega}} \times \hat{\mathbf{p}}}$, and $\hat{\mathbf{M}}_{\perp}=\hat{\mathbf{M}} \times \hat{\mathbf{\Omega}}$.

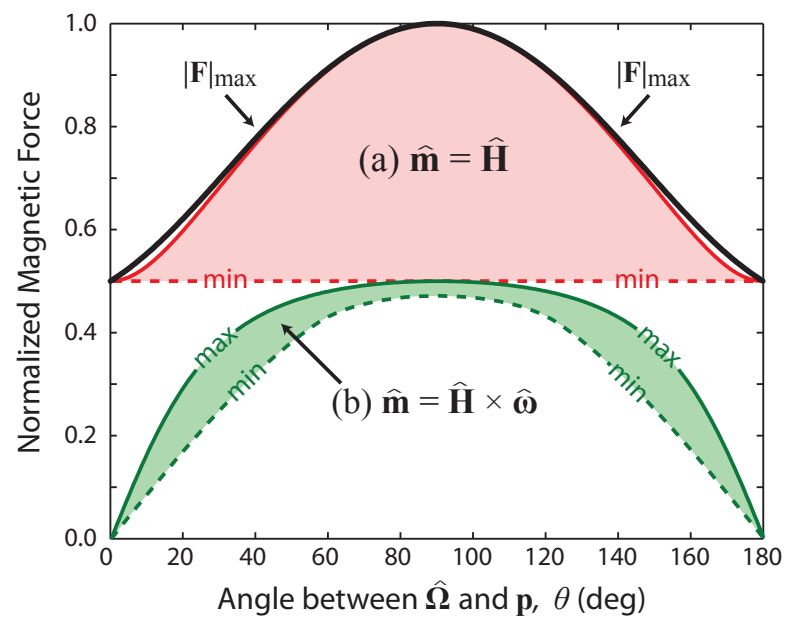

Fig. 2. The worst-case upper bound on force magnitude $|\mathbf{F}|_{\max }$, given by (7), is plotted alongside the most extreme range of forces possible when (a) the field $\mathbf{H}$ is rotated quasistatically such that the MAT dipole moment $\mathbf{m}$ is nearly always aligned with $\mathbf{H}$, and (b) the field is rotated at the MAT's instantaneous step-out frequency, making $\mathbf{m}$ trail $\mathbf{H}$ by $90^{\circ}$ and maximizing magnetic torque. There is a smooth transition between the step-out and quasistatic cases as the angle between $\mathbf{m}$ and $\mathbf{H}$ varies. Forces have been normalized by $2 \kappa$.

The instantaneous magnitude of (9) varies periodically as the RPM rotates. A numerical solution for the maximum value taken on by $|\mathbf{F}|$ over one RPM revolution, normalized by $2 \kappa$, is plotted as a function of $\theta$ in Fig. 2. For comparison, Fig. 2 also presents the smallest force magnitude over one RPM revolution when the MAT is operated quasistatically (i.e., $\hat{\mathbf{m}} \approx \hat{\mathbf{H}}$ ). Fig. 2 shows that the strongest force magnitude when the MAT is actuated such that the magnetic torque is maximized is always less than or equal to the weakest force magnitude when the MAT is actuated quasistatically. The best case occurs when the MAT is operated in an axial position $\left(\theta=0^{\circ}\right.$ or $\left.180^{\circ}\right)$, where $|\mathbf{F}|=0$ (this is demonstrated in [5]). The weakest force magnitude over one RPM revolution when the MAT is operated at maximum torque also appears in Fig. 2 for reference. Operating the MAT such that $\tau$ is maximized clearly minimizes $|\mathbf{F}|$.

The instantaneous direction of the magnetic force $\mathbf{F}$ when $\tau$ is maximized, given by (9), varies periodically while the RPM rotates. If the change in MAT position $\mathbf{p}$ relative to the center of the RPM is small over one revolution of the RPM (e.g., if the RPM position is updated to keep $\mathbf{p}$ constant), then the average of (9) per RPM revolution can be used to find the average contribution of the magnetic force to MAT propulsion. If the angle $\phi$ is used to parameterize the rotation of $\hat{\mathbf{M}}$ about the axis $\hat{\Omega}$, then the average of (9) over one revolution of the RPM is given by

$$
\mathbf{F}_{\text {avg }}=\frac{1}{2 \pi} \int_{0}^{2 \pi} \mathbf{F} \mathrm{d} \phi .
$$

After parameterizing $\hat{\mathbf{M}}$ with the angle $\phi$ and then integrating (9) over one complete revolution of $\phi$, we find

$$
\mathbf{F}_{\text {avg }}=\frac{2 \kappa}{3 \pi|\tilde{\mathbf{p}}|}\left(\mathrm{K}(k)+\left(3|\tilde{\mathbf{p}}|^{2}-1\right) \mathrm{E}(k)\right) \hat{\mathbf{d}}
$$


where $k=3|\tilde{\mathbf{p}}|^{2} /\left(1+3|\tilde{\mathbf{p}}|^{2}\right)$, and $\mathrm{K}(k)$ and $\mathrm{E}(k)$ are complete elliptic integrals of the first and second kinds, respectively. Note that when the MAT is in an axial position, where $\theta=0^{\circ}$ or $180^{\circ},|\tilde{\mathbf{p}}|=0$ and (11) breaks down. In this case, it can be verified using (9) that $|\mathbf{F}|=0$ for all time.

Equation (11) shows that $\mathbf{F}_{\text {avg }}$ always lies in the $\hat{\mathbf{d}}$ direction for every MAT position $\mathbf{p}$. Since $\hat{\mathbf{d}}$ is perpendicular to $\hat{\mathbf{p}}$ by definition, there is no attractive component of $\mathbf{F}_{\text {avg }}$ in the direction of the RPM (i.e., in the direction of $-\hat{\mathbf{p}}$ ). This phenomenon enables a MAT to be actuated away from the RPM even at close range, where the attractive magnetic force would typically be stronger than the propulsive force generated by the MAT (if the MAT were not being actuated such that the magnetic torque was maximized). This is demonstrated in a radial position in [6]. Averaging the magnetic force over one RPM revolution hides the instantaneous behavior of the force. If the time-scale of MAT motion is much less than that of the RPM (i.e., the RPM rotates quickly and the MAT moves slowly), then (11) becomes a good approximation of the net MAT behavior.

\section{EXPERIMENTAL Results \& Discussion}

The RPM is positioned using a Yaskawa Motoman MH5 6-DOF robotic manipulator (Fig. 3(a)). The RPM used in this paper consists of a cylindrical $25.4 \mathrm{~mm}$ diameter, $25.4 \mathrm{~mm}$ long, Grade-N42, diametrically magnetized (i.e., along the diameter) $\mathrm{NdFeB}$ permanent magnet driven by a Maxon $24 \mathrm{~V}$ A-Max Brushless DC motor with an Advanced Motion Controls servo control drive and amplifier. The point-dipole model (1) exactly predicts the field generated by a spherical permanent magnet, and is an approximation for every other geometry, which becomes more accurate with increasing distance. In [7], we found that (1) models the field generated by the RPM accurately at typical actuation distances and the RPM dipole moment $|\mathbf{M}|$ is $12.7 \mathrm{~A} \cdot \mathrm{m}^{2}$. Magnet geometries can be adjusted to improve the approximation of their field by the point-dipole model [12].

The RPM was used to actuate a threaded capsule-shaped MAT (Fig. 3(c)), which is a prototype of models that have been proposed to replace the currently passive capsule cameras used for endoscopic procedures in the human gastrointestinal system [2]. The capsule MAT is $24.0 \mathrm{~mm}$ long, $11.7 \mathrm{~mm}$ in diameter, and contains a $6.35 \mathrm{~mm}$ cubic Grade-N52 NdFeB magnet positioned at the device's centerof-gravity, with the dipole moment $\mathbf{m}$ oriented perpendicular to the capsule's principal axis and with magnitude $|\mathbf{m}|$ found experimentally to be $0.34 \mathrm{~A} \cdot \mathrm{m}^{2}$. The thread of the capsule MAT has a pitch of $34.6 \mathrm{~mm}$ and is $1.11 \mathrm{~mm}$ deep; this geometry is very similar to that presented in [13] as being optimal for propulsion on a thin film of mucous.

To verify the bounds presented in Fig. 2, we measured the force exerted by the RPM upon a permanent magnet of the same grade and geometry as that used in the capsule MAT, using an ATI Nano17 force/torque sensor (Fig. 3(b)). The RPM was placed in two different positions relative to the small permanent magnet, which are shown in Fig. 4(a) and 4(b), at a distance of $90.0 \mathrm{~mm}$, and rotated several

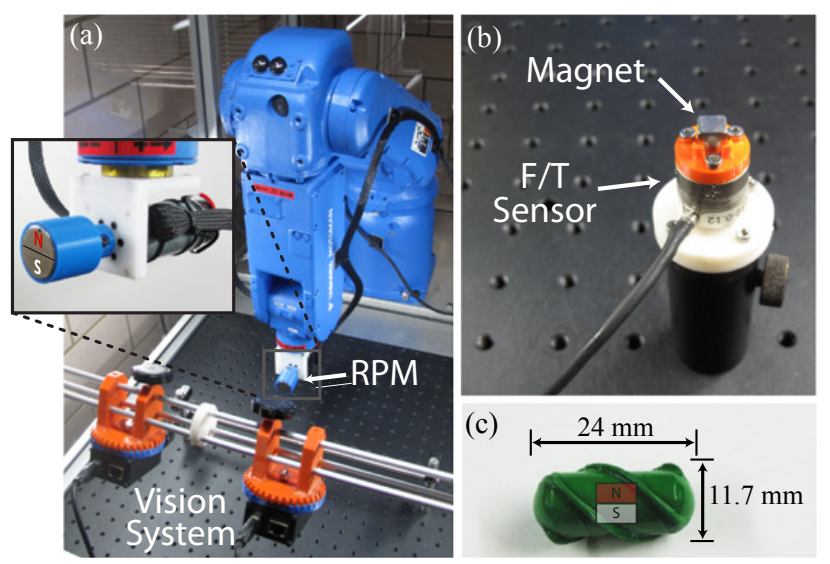

Fig. 3. The Yaskawa Motoman MH5 6-DOF robotic manipulator (a) is used to position the RPM for experiments. The setup containing the ATI Nano17 6-DOF force/torque sensor, used to obtain the results for Fig. 4, is shown in (b). The threaded capsule MAT, used to obtain the results in Fig. 6 , is shown in (c).

revolutions. Figs. 4(c) and 4(d) show the measured and predicted force by (5) at both positions. The worst-case force magnitude bound (7) and the minimum and maximum bounds for the case when torque is minimized (i.e., when a MAT is actuated quasistatically), corresponding to Fig. 2(a), and when the torque is maximized (i.e., when a MAT is actuated at its step-out frequency), corresponding to Fig. 2(b), are plotted as well. Because the magnet attached to the force sensor does not rotate with the field, the points on Figs. 4(a) and 4(b) where the bounds presented in Figs. 2(a) and 2(b) apply are in the peaks and troughs, respectively. Clearly, (5) is an accurate model of the magnetic force applied by the RPM on the MAT's permanent magnet.

When actuated such that the magnetic torque is always maximized, (11) shows that the time-averaged force over one RPM revolution applied to a MAT always points in a direction perpendicular to $\hat{\mathbf{p}}$ and no component of the averaged force attracts the MAT in the direction of the RPM. When the time-scale of MAT motion is slower than RPM rotation, then (11) predicts that, because there is no attractive component of average force, the contribution of the attractive force to MAT motion in the direction of the RPM should diminish when the magnetic torque is maximized in any MAT position. To demonstrate this phenomenon, we actuated the threaded MAT through a $12.7 \mathrm{~mm}$ inner-diameter PVC lumen (lightly lubricated with personal lubricant jelly) in two different positions where the RPM leads and trails the MAT, shown in Fig. 5(a) and 5(b), respectively. In both positions, the RPM is positioned $150.0 \mathrm{~mm}$ away from the MAT and $\hat{\Omega}$ is set so that $\hat{\boldsymbol{\omega}}$ is parallel to the lumen. The RPM positioned using the robotic manipulator and a stereo vision system (described in [7]), to maintain the relative position between the MAT and RPM as the MAT travels down the lumen. Still frames of the capsule MAT actuated in the lumen at $2.7 \mathrm{~Hz}$ in both positions are shown in Fig. 5(a) and 5(b).

The resulting MAT forward velocity at both MAT positions, when the angular velocity of the applied magnetic field 

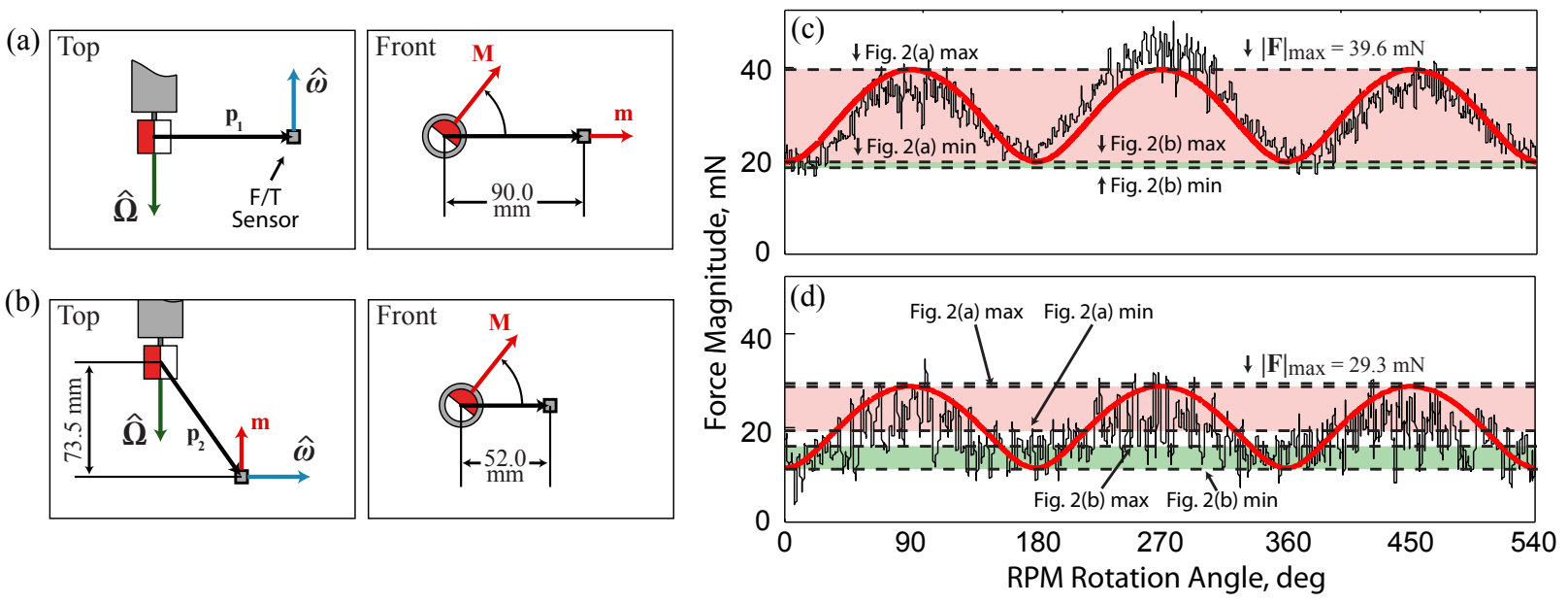

Fig. 4. The measured magnetic force (and the prediction by (5)) applied to a stationary permanent magnet as the RPM rotates, when positioned as in (a) and (b), is shown in (c) and (d), respectively. In both positions, the permanent magnet is positioned $90.0 \mathrm{~mm}$ from the RPM center and its dipole moment $\mathbf{m}$ is arranged to be perpendicular to $\hat{\boldsymbol{\omega}}$. The conservative force bound $|\mathbf{F}|_{\text {max }}$, given by (7), is shown in (c) and (d), along with the minimum and maximum force magnitude bounds when a MAT is actuated quasistatically (Fig. 2(a)) and such that torque is maximized (Fig. 2(b)). The peaks of (c) and (d) occur when $\mathbf{m}$ is parallel to $\hat{\mathbf{H}}$, where Fig. 2(a) applies, and the troughs occur when $\mathbf{m}$ is parallel to $\hat{\mathbf{H}} \times \hat{\boldsymbol{\omega}}$, where Fig. 2(b) applies. The force was measured using an ATI Nano17 6-DOF force/torque sensor (Fig. 3(b)) with $3.13 \mathrm{mN}$ sensing resolution, and filtered with a Butterworth filter.

is set at increasing percentages of the MAT's instantaneous step-out frequency (experimentally measured to be near $4.6 \mathrm{~Hz}$ ), is shown in Fig. 6(a), along with the percent speedup of the RPM-leading position over the RPM-trailing position shown in Fig. 6(b). Each data point in Fig. 6(a) is the average velocity of five trials and the error bars represent the full range of all trials. Fig. 6(b) shows the "speedup," defined as the ratio of the average MAT velocity in the leading position to that in the trailing position. When the applied field's angular velocity is slow compared to the step-out frequency, the applied magnetic torque is small, which makes the MAT dipole moment $\mathbf{m}$ nearly aligned with the applied field. In this configuration, the magnetic force primarily attracts the MAT toward the RPM. The attractive force contributes significantly to the MAT's forward velocity in the RPM-leading position, and significantly hinders the MAT in the RPM-trailing position. As the field's angular velocity nears the step-out frequency, the applied magnetic torque increases until it is maximized at $4.6 \mathrm{~Hz}$. In this configuration, no component of the time-averaged magnetic force lies in the direction of the RPM and the magnetic force contributes much less to the MAT's forward velocity in both positions, dramatically reducing the speedup of the RPMleading position over the RPM-trailing position.

In general, actuating MATs in RPM-leading positions produces the fastest MAT velocity toward the desired direction of travel and the RPM can be rotated at slower speeds to generate the same results compared to RPM-trailing positions. In addition, we find that RPM-leading positions tend to produce robust MAT actuation against naturally occurring disturbances; when the MAT steps out of synchronization due to an intermittent increase in friction, the resulting attractive force assists the MAT toward the desired direction of travel until synchronization can be regained. When possible, given any physical constraints imposed on the placement of the
RPM (e.g., to avoid collision with a patient), MATs should be actuated in RPM-leading positions. As illustrated herein, however, the influence of the attractive magnetic force on MAT actuation diminishes near step-out.

When the MAT is actuated at its step-out frequency as described herein, small changes in friction are enough to cause the MAT to step out of synchronization with the rotating magnetic field. For robustness, a MAT should be operated just below its step-out frequency or a control loop should be closed around the MAT's rotation [14]. There is a smooth transition between the case where the MAT is actuated at step-out and the MAT is actuated quasistatically on Fig. 2. Therefore, if the MAT rotates below its step-out frequency, then the minimum and maximum applied force magnitudes fall between the step-out and quasistatic cases.

If the MAT is desired to rotate at a specific frequency $|\boldsymbol{\omega}|_{d}$ while maintaining the magnetic force magnitude below some threshold, then there always exists some MAT position that can achieve both. One simple approach to finding an appropriate position is to first adjust the MAT-RPM separation distance to make $|\boldsymbol{\omega}|_{\text {so }}=|\boldsymbol{\omega}|_{d}$, and then select the appropriate angle $\theta$ (see Fig. 1) to ensure the magnetic force never exceeds the desired threshold according to Fig. 2. As the force threshold decreases, the necessary RPM position will approach an axial position. If the necessary position cannot be achieved due to geometric constraints (e.g., a patient's body) then the desired speed may not be achievable.

Aside from actively actuating the capsule to reduce the applied magnetic force, scaling the RPM in size can also reduce the magnetic force. If each dimension of the RPM is scaled by a factor $s$ (i.e., the volume and the dipole moment scales as $s^{3}$ ), then the RPM's magnetic field scales homothetically, meaning that the field measured at the position $s \mathbf{p}$, is the same as that of an unscaled RPM measured at the position $\mathbf{p}$. The force applied by a scaled RPM scales 
(a)

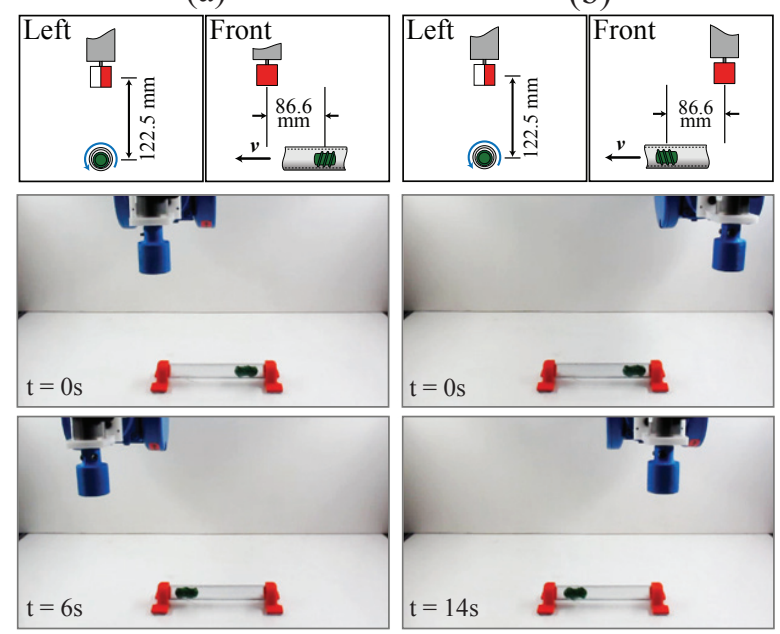

Fig. 5. The capsule MAT is actuated down the lumen in a position (a) where the RPM leads the MAT, and in a position (b) where the RPM trails the MAT. In both cases, the distance between the MAT and RPM is maintained at $150.0 \mathrm{~mm}$, and the RPM's position relative to the MAT is maintained using the robotic manipulator and stereo vision system shown in Fig. 3(a). Still images showing the MAT actuated with an RPM rotation speed of 2.7 $\mathrm{Hz}$ are shown as well.

as $s^{-1}$ when measured at $s \mathbf{p}$ [7], which implies that the influence of magnetic force will drop off while the available magnetic torque remains unchanged while actuating a MAT at the position $s \mathbf{p}$. The permanent magnet placed within our threaded capsule MAT is a $6.35 \mathrm{~mm}$ cube. Due to the electronics, batteries, and imaging equipment that must be fit into the small form-factor of a capsule endoscope, it is likely that a magnetic capsule endoscope will contain a smaller magnet in practice. The applied magnetic force and torque remain unchanged when scaling down the MAT's magnet by the factor $s^{-1}$ provided the RPM scales as $s$.

\section{CONCLUSION}

It is desirable to reduce the attractive force acting between a magnetically actuated tool (MAT) and its rotating permanent magnetic (RPM) actuator to prevent trauma when the MAT is an in vivo medical device, and when the RPM position generates attractive forces that hinder MAT locomotion. In this paper, we have presented a generalization of the force reduction strategies that were originally developed exclusively for use in axial and radial MAT positions to any MAT position. We find that when the MAT is dynamically actuated such that the applied magnetic torque is maximized, the instantaneous magnitude of the applied magnetic force is reduced compared to when the MAT is actuated quasistatically, and no component of the time-averaged magnetic force attracts the MAT to the RPM. We have provided experimental demonstration that validates and illustrates the force reduction strategies presented.

\section{REFERENCES}

[1] B. J. Nelson, I. K. Kaliakatsos, and J. J. Abbott, "Microrobots for minimally invasive medicine," Annu. Rev. Biomed. Eng., vol. 12, pp. $55-85,2010$ (a)

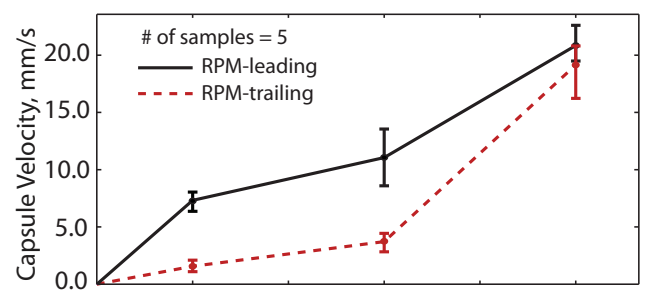

(b)

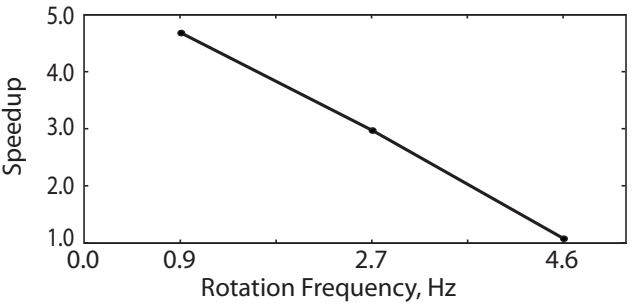

Fig. 6. The MAT forward velocity when actuated in the RPM-leading (Fig. 5(a)) and RPM-trailing (Fig. 5(b)) positions is shown plotted in (a) as a function of the RPM rotation frequency. Each data point is an average of five trials and the error bars represent the data range at each frequency. The "speedup," defined as the ratio of the trial-average MAT velocity in the RPM-leading position to that in the RPM-trailing position, is shown in (b)

[2] J. L. Toennies, G. Tortora, M. Simi, P. Valdastri, and R. J. Webster III, "Swallowable medical devices for diagnosis and surgery: the state of the art," J. Mech. Eng. Sci., vol. 224, no. 7, pp. 1397-1414, 2010.

[3] M. P. Kummer, J. J. Abbott, B. E. Kratochvil, R. Borer, A. Sengul, and B. J. Nelson, "Octomag: An electromagnetic systems for 5-DOF wireless micromanipulation," IEEE Trans. Robot., vol. 26, no. 6, pp 1006-1017, 2010

[4] G. Ciuti, P. Valdastri, A. Menciassi, and P. Dario, "Robotic magnetic steering and locomotion of capsule endoscope for diagnostic and surgical endoluminal procedures," Robotica, vol. 28, no. 2, pp. 199 $207,2010$.

[5] T. W. R. Fountain, P. V. Kailat, and J. J. Abbott, "Wireless control of magnetic helical microrobots using a rotating-permanent-magnet manipulator," in Proc. IEEE Int. Conf. Robotics and Automation, 2010, pp. $576-581$

[6] A. W. Mahoney and J. J. Abbott, "Managing magnetic force applied to a magnetic device by a rotating dipole field," Appl. Phys. Lett., vol. 99 , no. 134103 , pp. $1-3,2011$.

[7] A. W. Mahoney, D. L. Cowan, K. M. Miller, and J. J. Abbott, "Control of untethered magnetically actuated tools using a rotating permanent magnet in any position," in Proc. IEEE Int. Conf. Robotics and Automation, 2012, pp. 3375-3380.

[8] S. Yim and M. Sitti, "Design and rolling locomotion of a magnetically actuated soft capsule endoscope," IEEE Trans. Robot., vol. 28, no. 1, pp. 183-194, 2012.

[9] G. Lien, C. Liu, J. Jiang, C. Chuang, and M. Teng, "Magnetic control system targeted for capsule endoscopic operations in the stomach: Design, fabrication, and in vitro and ex vivo evaluations," IEEE Trans. Biomed. Eng., vol. 59, no. 7, pp. 2068-2079, 2012.

[10] M. T. Hou, H.-M. Shen, G.-L. Jiang, C.-N. Lu, I.-J. Hsu, and J. A. Yeh, "A rolling locomotion method for untethered magnetic microrobots," Appl. Phys. Lett., vol. 96, no. 024102, pp. 1-3, 2010.

[11] H. Keller, A. Juloski, H. Kawano, M. Bechtold, A. Kimura, H. Takizawa, and R. Kuth, "Method for navigation and control of a magnetically guided capsule endoscope in the human stomach," in IEEE Int. Conf. Biomedical Robotics and Biomechatronics, 2012, pp. 859-865.

[12] A. J. Petruska and J. J. Abbott, "Optimal permanent-magnet geometries for dipole field approximation," IEEE Trans. Magn., vol. 49, no. 2, pp. 811-819, 2013.

[13] K. Ikeuchi, K. Yoshinaka, and N. Tomita, "Low invasive propulsion of medical devices by traction using mucus," Wear, vol. 209, pp. 179183, 1997.

[14] K. M. Miller, A. W. Mahoney, T. Schmid, and J. J. Abbott, "Proprioceptive magnetic-field sensing for closed-loop control of magnetic capsule endoscopes," in IEEE/RSJ Int. Conf. Intelligent Robots and Systems, 2012, pp. 1994-1999. 